\title{
Evaluation of Oxidative DNA Damage and Its Relation with Anthropometric Indices in Healthy Adolescents
}

\author{
Farzaneh Bagherzadeh $^{1}$, Mehrangiz Ebrahimi-Mameghani ${ }^{1}$, Mohammad Asghari Jafarabadi ${ }^{2}$, \\ Zahra Aslani ${ }^{3}$, Sare Edalati ${ }^{1}$ \\ ${ }^{1}$ Department of Nutrition in Society, Nutrition Faculty, Tabriz University of Medical Sciences, Tabriz, Iran \\ ${ }^{2}$ Medical Education Research Center, Department of Statistics and Epidemiology, Faculty of Health, Tabriz University of Medical Sciences, \\ Tabriz, Iran \\ ${ }^{3}$ Nutrition and Endocrine Research Center, and Obesity Research Center, Research Institute for Endocrine Sciences, Shahid Beheshti \\ University of Medical Sciences, Tehran, Iran
}

Email address:

ebrahimimamgani@tbzmed.ac.ir (M. Ebrahimi-Mameghani)

\section{To cite this article:}

Farzaneh Bagherzadeh, Mehrangiz Ebrahimi-Mameghani, Mohammad Asghari Jafarabadi, Zahra Aslani, Sare Edalati. Evaluation of Oxidative DNA Damage and Its Relation with Anthropometric Indices in Healthy Adolescents. International Journal of Nutrition and Food Sciences. Special Issue: Functional Foods and Nutraceuticals for Management of Type 2 Diabetes. Vol. 3, No. 2-2, 2015 , pp. 21-25. doi: 10.11648/j.ajbio.s.2015030202.11

\begin{abstract}
Reactive oxygen species (ROS) and oxidative stress (OS) have important role in prevention, initiation and progression of chronic diseases from early childhood, but OS status in healthy young subjects and appropriate methods for its measurement have been remained unclear. Evaluation of urinary biomarker set for OS has not been well documented in Iran. In this study, evaluation of OS from urinary biomarker in healthy adolescents and its relevance to anthropometric index were investigated. Forty- hundred students aged 13 and 19 years old participated in this study. 8-hydroxy-2'-deoxyguanosine (8$\mathrm{OHdG}$ ) as marker of oxidative DNA damage was measured in adolescents and also its relation with age, sex and body mass index was studied. Subjects were sampled by multistage cluster sampling. The average ratio of 8 -OHdG to creatinine was 4.7 4.1. No significant difference of $8-\mathrm{OHdG}$ was observed between boys and girls in this study.
\end{abstract}

Keywords: Oxidative Stress, Oxidative DNA Damage, Adolescents, Body Mass Index, Age, Sex

\section{Introduction}

Reactive oxygen species (ROS) are cell metabolites specially mitochondria. Only little amount of ROS is engaged in cell signaling and defending against pathogen parameters invasion, whereas they are more considered for damaging organs. Cells have antioxidant system for inhabitation and removal of these materials and there is equilibrium between production and neutralization of ROS in normal physiological status. Oxidative stress (OS) is occurred when production process of ROS is accelerated or when the mechanisms involved in keeping cell environment are disordered [1, 2]. OS is more likely along with pathological phenomena such as aging, arteriosclerosis, high blood pressure, renal disorders, changes in the immune system, damage to revascularization cycle, nerve damage, cancers and destruction and inflammation conditions [3, 4, $5,6]$. OS can simultaneously result damage in biomolecules including lipids, proteins, nucleic acids and carbohydrates $[7,8]$. The direct measurement of ROS is difficult because of its short half-life; Therefore OS biomarkers are measured as oxidative process result. Assessment of OS biomarkers is various. One of OS biomarkers is 8-hydroxy-2'deoxyguanosine $(8-\mathrm{OHdG})$-base guanine oxidation- is secreted to urine $[9,11]$. It is considered as one of the most sensitive indicators of oxidative cell damage because of its non-invasiveness and high sensitivity. This index is a useful maker for evaluation of cardiovascular disease $\left(\mathrm{CVD}_{\mathrm{s}}\right)$ and inflammatory status in patients with high blood pressure. In addition, it helps to identify individuals at risk of cancer and early prediction of risks associated with lifestyle-related diseases. [13, 14,15]. Recent observations have shown that OS is related with childhood diseases and conditions that disease has not been appeared yet. Oxidative injuries in pathological conditions have more serious consequences in children than in adults, due to tissue growth and adaptation to 
physical growth and survival $[16,17,18]$. Thus preventing from oxidative damage, especially in young people is more considered today [19]. Nonetheless, the level of oxidative stress in healthy children and adolescents has not been known comprehensively yet. Discharge measurement of8$\mathrm{OHdG}$ in urine may reflect systemic oxidative stress [12] and also this index has unique ability to assess DNA damage in pathophysiology of metabolic risk factors and atherosclerosis $[10,20]$.However, the functional significance of a method is its convenience and reliability. Recently, 8-OhdG is much used as an indicator of oxidative stress [21]. Despite this, few studies have been conducted on the $8-\mathrm{OHdG}$ in healthy adolescents [22]. As the evaluation of OS biomarker by urinary sampling has not been done in apparently healthy adolescents in Iran and according to convenience and reliability of urine sampling, we therefore conducted this study to evaluate $8-\mathrm{OHdG}$ and its relation to age, sex and body mass in healthy adolescences.

\section{Materials and Methods}

Methods are presented as blow:

\subsection{Study Population}

This trial was a cross- sectional study. Researchers entered to high schools after obtaining necessary permits from Education of Alborz in Iran. This trial was done between April and May 2013. The protocol of this study was approved by Ethics Committee of Tabriz University of Medical Sciences. All of the sampling was performed between 10-12 AM. Fortyhundred healthy adolescents were participated in 5 areas of Alborz Province by multistage cluster sampling. Participants aged 13-19 and were apparently healthy. They included 78 boys and 62 girls. No significant difference in age was found between boys and girls. The subjects with renal or hepatic disorders, diabetes, cancer, CVDs, hypertension and hypothyroid were excluded from the study. Students were provided with written informed consent from their parents. None of the participants used any drugs or antioxidants during the last few days. Students who have used some supplements during the last 6 months were excluded from the study.

\subsection{Anthropometric and Biochemical Measurements}

Anthropometric measurements including weight, height and waist circumference were measured upon arrival. Weight was measured with a scale (Seca, Hamburg, Germany) while the subjects were minimally clothed and without shoes. Height was measured by Seca tape meter with $0.5-\mathrm{cm}$ accuracy, in standing position without shoes. Body mass index (BMI) was calculated as follow: weight /square of height $\left(\mathrm{Kg} / \mathrm{m}^{2}\right)$. Metabolic Equivalents (MET) questionnaire was used and physical activity level was into 9 rows, based on their intensity. Rows included 0.9, 1, 1.5, 2, 3, 4, 5,6 and upper than 6 respectively [23]. The time spends for each physical activity has multiplied by the METs value of that activity [23]. Then, all obtained data (MET.H) were added (day/MET.H). Urine samples were taken randomly and the clear supernatant was removed after 10 minutes of centrifuge. Density of $8-\mathrm{OHdG}(\mathrm{ng} / \mathrm{ml})$ in urine samples was measured by a competitive kit (Cusabio Biotech, kn: CSB-E10140h, Japan) [8]. The examiners were blinded to the clinical and laboratory results. Urinary creatinine was used for correction of urinary $8-\mathrm{OHdG}$ concentration. Fixed correlation has been reported between the random level of urine and $8-\mathrm{OHdG}$ discharge in 24 hours [24].

\subsection{Statistical Analysis}

The experimental data was analyzed by statistical package for the social sciences (SPSS software, version16; SPSS Inc., Chicago, IL, USA).Quantitative data was presented as mean $\pm \mathrm{SD}$ and qualitative data as frequencies and percentages. Two groups (boys and girls) were compared by independent t-test. Partial correlation was used by adjustment of confounders including physical activity and exposure to cigarette. Also, $\mathrm{p}$ - value $<0.05$ was considered significant.

\section{Results}

Subjects' demographics are shown in Table 1. The average height of boys was significantly higher than girls. Age, weight, BMI (body mass index) and physical activity of subjects had not significant difference. In order to avoid the variety of 8-OHdGindexes and changes of water in use, it is recommended to use urine creatinine [24], therefor the result was reported in 2 forms including $\mathrm{ng} / \mathrm{mg}$ urine and $\mathrm{ng} / \mathrm{mL}$ density of urine creatinine in both samples of boys and girls (Table 2). There was no significant difference of8-OHdG $(\mathrm{ng} / \mathrm{ml})$ between boys and girls. No significant relation was observed between $8-\mathrm{OHdG}$ index $(\mathrm{ng} / \mathrm{ml})$, age, weight, BMI and physical activity (Table 3). Furthermore, in this study participants were divided in four age categories for reporting the effect of age on the index (8-OHdG) (Table 4).

Table 1. Subjects' characteristic participated in study.

\begin{tabular}{lllll}
\hline $\mathbf{P}$ & Boys $(\mathbf{n}=\mathbf{7 8})$ & Girls $(\mathbf{n}=\mathbf{6 2})$ & All $(\mathbf{n}=\mathbf{1 4 0})$ & Variables \\
\hline 0.5 & $15.9 \pm 1.9$ & $15.5 \pm 1.3$ & $15.7 \pm 1.7$ & Age $($ year $)$ \\
0.07 & $50.2 \pm 10.7$ & $46.1 \pm 16.5$ & $48.4 \pm 13.7$ & MET( MET-h/day) \\
0.06 & $59.9 \pm 14.3$ & $55.6 \pm 13.2$ & 58.00 & Weight $(\mathrm{Kg})$ \\
$<0.01$ & $159 \pm 19$ & $155 \pm 13$ & $164.4 \pm 10.2$ & Height $(\mathrm{cm})$ \\
0.3 & $21 \pm 3.6$ & $21.3 \pm 4.3$ & $21.3 \pm 3.9$ & BMI $\left(\mathrm{kg} / \mathrm{m}^{2}\right)$ \\
0.7 & $37.2 \%$ & $40.3 \%$ & $61.6 \%$ & Exposure to cigarette \\
\hline
\end{tabular}

Data are mean \pm SD unless stated otherwise. MET, metabolic equivalents; BMI, body mass index ;

Calculated by using independent t-test 
Table 2. Urinary levels of 8-OHdG in healthy young peoples ${ }^{c}$.

\begin{tabular}{|c|c|c|c|c|}
\hline Variables & All (140) & Girls $(n=62)$ & Boys $(n=78)$ & $\mathbf{P}^{\mathbf{d}}$ \\
\hline 8-OHdG(ng/mL) & $60.1 \pm 4.9$ & $5.3 \pm 4.5$ & $6.6 \pm 5.2$ & 0.1 \\
\hline 8-OHdG(ng/mgcr) & $4.7 \pm 4.1$ & $4.5 \pm 3.9$ & $4.9 \pm 4.1$ & 0.5 \\
\hline
\end{tabular}

8-OHdG, 8-hydroxy-2'-deoxyguanosine;

Data are mean $\pm \mathrm{SD}$;

Calculated by using independent t-test

Table 3. Correlations between urinary $8-O H d G$ and all the measured variables.

\begin{tabular}{llllll}
\hline \multirow{2}{*}{ Variables } & All $(\mathbf{n = 1 4 0 )}$ & & boy(n=78) & \multicolumn{2}{c}{ girl(n=62) } \\
\cline { 2 - 6 } & $\mathbf{r}$ & P value & R & P value & R \\
\hline Age(year) & -0.08 & 0.3 & -0.05 & 0.7 & -0.2 \\
BMI $\left(\mathrm{kg} / \mathrm{m}^{2}\right)$ & -0.03 & 0.7 & 0.03 & 0.8 & -0.06 \\
MET( MET-h/day $)$ & 0.00 & 0.9 & 0.2 & 0.2 & -0.2 \\
\hline
\end{tabular}

$\mathrm{r}=$ partial correlation coefficient

Table 4. Age-related changes in urinary levels $8-O H d G$ (ng/mg creatinine)

\begin{tabular}{|c|c|c|c|c|}
\hline & $13-15 y$ & $15-16 y$ & $16-17 y$ & $17-19 y$ \\
\hline $\mathrm{N}$ & 37 & 34 & 39 & 30 \\
\hline Minimum & 0.8 & 0.6 & 0.9 & 0.6 \\
\hline 25 percentile & 1.6 & 1.3 & 1.9 & 1.6 \\
\hline 50 percentile & 3.6 & 3.7 & 4.2 & 3.2 \\
\hline 75 percentile & 8.1 & 6.3 & 7.1 & 5.8 \\
\hline Maximum & 26.6 & 16.7 & 15.4 & 16.5 \\
\hline
\end{tabular}

Calculate data frequency.

Data are mean in age groups.

\section{Discussion}

OS caused by the difference between ROS production and antioxidant activity. Imbalance between oxidant and antioxidant status has been proposed as a leading health concern in children and adolescents [25]. Meanwhile, no study has measured $8-\mathrm{OHdG}$ index in Iran. In some published studies, it has been reported that oxidative stress plays a key role in the development of biological defaults, including $\mathrm{CVD}_{\mathrm{S}}$, metabolic diseases, cancer, and aging disorders [7]. Primary and secondary preventive actions against oxidative damage, especially in young people are important. Moreover, the use of antioxidants as a new therapeutic approach for diseases associated with increased oxidative stress has been proposed. Investigating the role of OS in children needs information about the status of OS in young people. Whereas, there are only a few reports about the status of DNA OS in children and adolescents. In these studies few numbers of people with low age range are considered. In most studies blood samples were collected. In most studies blood samples were collected and reduced / oxidized glutathione, glutathione peroxidase and glutathionereductase activity, selenium, vitamins, antioxidants, reduced / oxidized coenzyme Q10, thiobarbituric acid, superoxide and catalase activities were analyzed [26, 27, 28]. In addition, in most studies OS markers were evaluated in protein and fat according to intervention method. The aim of most studies was not to determine and evaluate the OS and the sample size and age range were determined. According to researchers, only two studies have been conducted to evaluate. Kauffman et al measured F2 isoprostane of urine in 342 children under
7 years old [26]. Tamura et al released that there was inverse relationship between age, 8-OHdG, nitrite / nitrate, and pentosidine in 100 children [18]. In this study age rang was 1-21 and due to high physiological growth in this age range; there was a logic correlation between age and 8-OHdG. The average of $8-\mathrm{OHdG}$ was 9.3 in Japanese adolescents, However it was 4.76 in present study. This could be because of differences in diet in the community [18]. Atabaket al showed that radical proxy level (another marker of OS) was higher in obese children compared with non-obese Tork children [29]. Urine collection is a rapid, non-invasive and functional method, and therefore particularly easy to perform in children. Collection of random urine sample is better than 24 hours according to its ability to run. Due to variety of water intake, random urine is standardized by correction of creatinine. Therefore, it is assumed that the urinary levels reflected fixed status in participant. It seems that one of major aims of OS free radicals attack is nuclear DNA and mitochondria. Among Purine and pyrimidine bases, Guanine is more susceptible to oxidation. The hydroxyl radical can attack to $\mathrm{C}-8$ position guanine and generated an oxidation product, 8 -hydroxydeoxyguanosine $(8-\mathrm{OHdG})[30,4]$. This compound is released as a result of DNA repairing processes and excreted without being metabolized in the urine. Mutagenic property of $8-\mathrm{OHdG}$ is well known. This compound is an important biomarker of cellular OS and considered as DNA repairing product. As $8-\mathrm{OHdG}$ shows dynamic equilibrium between DNA oxidative damage and repairing speed of it, the measurement of this compound in urine is important in the evaluation of DNA damage in whole body $[10,31]$. 


\section{Conclusion}

In this study, results have showed that there are not significant changes in $8-\mathrm{OHdG}$ in 13-19 years old participants, which is consistent with other studies. In studies with infant participants, there were significant differences between the age groups and 8-OHdG compared with upper age group. As the subjects were healthy, there was seen correlation between body mass index and $8-\mathrm{OHdG}$. In short, we determine age-related changes of urinary marker of oxidative stress in adolescences. In this study, a normal amount was reported that may be useful to assess OS in diseases of young people. This non-invasive indicator can be efficient for future studies that will measure antioxidants on OS-related diseases. The present study has a few limitations because of the age range and food dietary.

\section{Acknowledgement}

We thank the Nutrition Research Center and Student Research Committee of Medical Sciences for their financial support. This article is provided from MSc. Authors appreciate the staff education Alborz Province, Iran for their sincere cooperation. The authors declare that there are no conflict of interest.

\section{References}

[1] Rahman I, Kelly F. Review Biomarkers in Breath Condensate: A promising New Non-invasive Technique in Free Radical Research. FRA. 2003;37(12):1253-66.

[2] Offord E, Van Poppel G, Tyrrell R. Markers of oxidative damage and antioxidant protection: current status and relevance to disease. FRA. 2000;33:S5.

[3] Nemeth I, Boda D. Xanthine oxidase activity and blood glutathione redox ratio in infants and children with septic shock syndrome. JIC. 2001;27(1):21-26.

[4] Tsukahara H, Sekine K, Uchiyama M, Kawakami H, Hata I, Todoroki Y, et al. Formation of advanced glycosylation end products and oxidative stress in young patients with type 1 diabetes. Pediatr. Res. 2003;54(3):419-24.

[5] Kaneko K, Taniguchi N, Tanabe Y, Nakano T, Hasui M, Nozu $\mathrm{K}$. Oxidative imbalance in idiopathic renal hypouricemia. Pediatr Neph. 2009;24(4):869-71.

[6] Tung EW, Winn LM. Valproic acid increases formation of reactive oxygen species and induces apoptosis in postimplantation embryos: a role for oxidative stress in valproic acid-induced neural tube defects. Mol. Pharmacol.. 2011;80(6):979-87.

[7] Granot E, Kohen R. Oxidative stress in childhood-in health and disease states. Clin Nutr. 2004;23:3-11.

[8] Toyokuni S, Tanaka T, Hattori Y, Nishiyama Y, Yoshida A, Uchida $K$, et al. Quantitative immunohistochemical determination of 8-hydroxy-2'-deoxyguanosine by a monoclonal antibody N45. 1: its application to ferric nitrilotriacetate-induced renal carcinogenesis model. Laboratory investigation; Lab Invest. 1997;76(3):365-74.
[9] Jang-Young K, Jun-Won L, YounMin-Soo A, Sung-Gyun A. Urinary Levels of 8-Iso-Prostaglandin F2 $\alpha$ and 8Hydroxydeoxyguanine as Markers of Oxidative Stress in PatientsWith Coronary Artery Disease. Korean Circ J. 2012;42:614-7.

[10] Valavanidis A, Vlachogianni T, Fiotakis C, C JESH. 8Hydroxy-2'-deoxyguanosine (8-OHdG): acritical biomarker of oxidative stress and carcinogenesis. 2009;27:120-39.

[11] Wu LL, Chiou C-C, Chang P-Y, Wu JT. Urinary 8-OHdG: a marker of oxidative stress to DNA and a risk factor for cancer, atherosclerosis and diabetics. Clinica Chimica Acta. 2004;339(1):1-9.

[12] Subash P, Gurumurthy P, Sarasabharathi A, Cherian K. Urinary 8-OHdG: a marker of oxidative stress to dna and total antioxidant status in essential hypertension with South Indian population. IJCB. 2010;25(2):127-32.

[13] Hwang E-S, Kim G-H. RETRACTED: Biomarkers for oxidative stress status of DNA, lipids , and proteins in vitro and in vivo cancer research. Toxicology. 2007;229(1):1-10.

[14] Sakano N, Wang D-H, Takahashi N, Wang B, Sauriasari R, Kanbara S, et al. Oxidative stress biomarkers and lifestyles in Japanese healthy people. JCBN2009;44(2):185.

[15] Halliwell B. Why and how should we measure oxidative DNA damage in nutritional studies? How far have we come? Am Clin Nutr. 2000;72(5):1082-7.

[16] Drury JA, Jeffers G ,Cooke RW. Urinary 8hydroxydeoxyguanosine in infants and children. FRA. 1998; 28(4): 423-8.

[17] Kaneko K, Kimata T, Tsuji S, Ohashi A, Imai Y, Sudo H, et al. Measurement of urinary 8-oxo-7,8-dihydro-2-deoxyguanosine in a novel point-of-care testing device to assess oxidative stress in children. Clinica Chimica Acta. 2012;413:1822-6.

[18] Tamura S, Tsukahara H, Ueno M. Evaluation of a urinary multi-parameter biomarker set for oxidative stress in children, adolescents and young adults. FreeRadic Res. 2006;40:1198205.

[19] Tsukahara H. Biomarkers for oxidative stress: clinical application in pediatric medicine. Curr Med Chem. 2007; 14:339-51.

[20] Piconi L, Quagliaro L, Ceriello A. Oxidative stress in diabetes. Clinical chemistry and laborator Med. 2003;41(9):1144-9.

[21] Kasai H, Kawai K, Li Y-s. Analysis of 8-OH-dG and 8-OHGua as biomarkers of oxidative stress. Genes and Environment. 2008;30(2):33-40.

[22] Tamura S, Tsukahara H, Ueno M, Maeda M, Kawakami H, Sekine K, et al. Evaluation of a urinary multi-parameter biomarker set for oxidative stress in children, adolescents and young adults. FRA. 2006;40(11):1198-205.

[23] Ainsworth BE, Haskell WL, Whitt MC, Irwin ML, Swartz AM, Strath SJ, et al. Compendium of physical activities: an update of activity codes and MET intensities. MSSE. 2000;32(9; SUPP/1):S498-S504.

[24] Poulsen HE, Loft S, Prieme H, Vistisen K, Lykkesfeldt J, Nyyssonen $\mathrm{K}$, et al. Oxidative DNA damage in vivo: relationship to age, plasma antioxidants, drug metabolism, glutathione-S-transferase activity and urinary creatinine excretion. FRA. 1998;29(6):565-71. 
[25] Chiavaroli V, Giannini C, De Marco S, Chiarelli F, Mohn A. Unbalanced oxidant-antioxidant status and its effects in pediatric diseases. Redox Report. 2011;16(3):101-7.

[26] Kauffman LD, Sokol RJ, Jones RH, Awad JA, Rewers MJ, Norris JM. Urinary F 2-isoprostanes in young healthy children at risk for type 1 diabetes mellitus. FRB M. 2003;35(6):551-7.

[27] Andreazza AC, Bordin DL, Salvador M. Thiobarbituric acid reactive substances, seric superoxide dismutase and catalase activities in healthy subjects. Clinica Chimica Acta. 2005; 362(1):192-4.

[28] Miles MV, Horn PS, Tang PH, Morrison JA, Miles L, DeGrauw T, et al. Age-related changes in plasma coenzyme Q 10 concentrations and redox state in apparently healthy children and adults. Clinica Chimica Acta. 2004; 347(1):13944.
[29] Atabek ME, Vatansev $\mathrm{H}$,Erkul I. Oxidative stress in childhood obesity. J PEM. 2004; 17(8): 1063-8.

[30] Evans MD, Dizdaroglu M, Cooke MS. Oxidative DNA damage and disease: induction, repair and significance. Mutation Research/Reviews in Mutation Research. 2004; 567(1): 1-61.

[31] Chiou C-C, Chang P-Y, Chan E-C, Wu T-L, Tsao K-C, Wu JT. Urinary 8-hydroxydeoxyguanosine and its analogs as DNA marker of oxidative stress: development of an ELISA and measurement in both bladder and prostate cancers. Clinica Chimica Acta. 2003; 334(1):87-94. 